\title{
A NOTE ON GROUP INVARIANT CONTINUA
}

\author{
WILLIAM J. GRAY
}

(Received 17 October 1966)

Let $(X, T, \pi)$ be a topological transformation group, where $X$ is a Hausdorff continuum. We will say that $X$ is irreducibly $T$-invariant if no proper subcontinuum of $X$ is $T$-invariant. Wallace, [6], has shown that if $T$ is abelian and $X$ is irreducibly $T$-invariant, then $X$ has no cut point; he then asked if this statement remains true if "abelian" is replaced by "compact". In this paper we answer this question in the affirmative, and prove a related result when $T$ satisfies a recursive property.

A general problem due to Wallace is as follows: Assume $T$ leaves an endpoint of $X$ fixed. Under what conditions on $X$ and $T$ does $T$ have another fixed point? This problem has been investigated by Wallace [8], Wang, [5], Chu, [1], and Gray, $[3,4]$. In Theorem 2, we show that if $X$ is locally connected, and $T$ is generated by a compact subgroup and a connected subgroup, then $T$ has another fixed point.

Departing from [7] slightly, we call a subcontinuum $C$ of $X$ a universal subcontinuum (USC) if given a subcontinuum $D$ of $X, D \cap C$ is a continuum. The intersection of arbitrarily many USC is again a USC. If $X-x=U \cup V$, where $U$ and $V$ are non-empty separated subsets of $X$ (hereafter referred to as a "separation of $X-x$ "), then $U \cup\{x\}$ is a USC. The property of being a USC is topological. The proofs of these statements are to be found in [7].

The terminology pertaining to transformation groups is taken from [2].

THEOREM 1. Let $(X, T, \pi)$ be a topological transformation group where $X$ is a Hausdorff continuum and one of the following conditions is satisfied:

(i) $T$ is compact,

(ii) $X$ is locally connected and $T$ is pointreise regularly almost periodic.

If $X$ is irreducibly $T$-invariant, then $X$ contains no cut point.

Proof. Assume that $X$ is irreducibly $T$-invariant. We make the following observations:

1. If $x \in X$, no proper $U S C$ of $X$ contains the orbit $T x$ of $x$.

For otherwise the intersection, $D$, of all USC which contains $T x$ is a proper subcontinuum of $X$ containing $T x$. We easily verify that that $D$ is $T$-invariant, and hence $X$ is not irreducibly $T$-invariant. 
2. If $X-x=U \cup V$ is a separation of $X-x$, then $U$ and $V$ both contain a cut point of $X$.

For by 1 , we cannot have $T x \subset U \cup\{x\}$, hence $T x \cap V \neq \emptyset$. Likewise $T x \cap U \neq \emptyset$. Since $T x$ contains only cut points of $X$, we have the desired result.

We now prove the theorem by contradiction! Assume $X$ contains a cut point. Let $y$ be a fixed non-cut point of $X$; for each cut point $x \in X$, choose a fixed separation $X-x=U_{x} \cup V_{x}$ of $X-x$ with $y \in U_{x}$ and let $H_{x}=V_{x} \cup\{x\}$. Order the collection of all such $H_{x}$ by inclusion and let $\mathscr{C}=\left\{H_{\alpha} ; \alpha \in I\right\}$ be a maximal totally ordered subcollection. Set $H=\cap\left\{H_{\alpha} ; \alpha \in I\right\} \neq \emptyset$.

If $H$ contains a cut point $x$ of $X$, let $X-x=U \cup V$ be any separation of $X-x$ with $y \in U$. Let $w$ be a cut point in $V$ and $X-w=W_{1} \cup W_{2}$ be any separation of $X-w$ with $y \in W_{1}$. If $\alpha \in I$ such that $x \neq x_{\alpha}$, then $x \in V_{\alpha}$, hence $U_{\alpha} \cup\left\{x_{\alpha}\right\} \subset U$. Then $V \cup\{x\} \subset V_{\alpha}$. Also, since $w \in V$, $U \cup\{x\} \subset W_{1}$, hence $W_{2} \cup\{w\} \subset V$. Thus if $x \neq x_{\alpha}$ for all $\alpha \in I, H_{x}$ is a proper subset of each $H_{\alpha}$. Otherwise, $H_{\omega}$ is a proper subset of each $H_{\alpha}$. Thus in any case $\mathscr{C}$ is not maximal. This contradiction shows that $H$ contains no cut point of $X$.

Assume $T$ is compact and let $x$ be any cut point of $X$. Since $H$ contains no cut points, $H \cap T x=\emptyset$. Since $T$ is compact, $X-T x$ is an open set containing $H=\cap\left\{H_{\alpha} ; \alpha \in I\right\}$. Therefore for some $\beta \in I$, we have $H_{\beta}=V_{\beta} \cup\left\{x_{\beta}\right\} \subset X-T x$. Then $T x \subset U_{\beta}$, and $U_{\beta} \cup\left\{x_{\beta}\right\}$ is a proper USC of $X$ containing $T x$, which contradicts 2 . This completes the proof in case (i).

Now assume (ii) holds. Let $X-x=U \cup V$ be a separation of $X-x$, and let $y$ be a cut point of $X$ with $y \in U$; by 1 ., there is a $t \in T$ such that $z=t y \in V$. Since $T$ is regularly almost periodic at $y$, it easily follows that there is a syndetic invariant subgroup $S$ of $T$ such that $S y \subset U$ and $S z \subset V$. Let $E(y, z)=\{w ; w$ separates $y$ and $z$ in $X\} \cup\{y, z\}$. It is clear that $S x \subset E(y, z)$. Since $X$ is locally connected, $E(y, z)$ is closed, thus $\overline{S x} \subset E(y, z)$ so that $\overline{S x}$ contains cut points alone. Let $K$ be a compact subset of $T$ such that $T=K S$. Then $K \overline{S x}$ is a compact set of cut points of $X$. We may complete the proof as in case (i).

The definition of an end point is that found in [5] namely: $e$ is an end-point of $X$ if $e$ does not separate $X$ and given any open set $U$ containing $e$, there exists $y \in U$ such that $X-y=P \cup Q, P$ and $Q$ separated, and $e \in P \leqq U$.

THEOREM 2. Let $(X, T, \pi)$ be a topological transformation group, where $X$ is a non-trivial locally connected Hausdorff continuum and $T$ is generated by a compact subgroup $C$ and a connected subgroup $K$. If $T$ leaves an end point e of $X$ fixed, then $T$ has another fixed point. 
Proof. Let $z$ be a non-cut point of $X$ other than $e$ and let $X-x=U \cup V$ be a separation of $X-x$ such that $e \in U$ and $C z \subset V$. If $p \in C z$, then $K p$ is a connected set of non-cut points of $X$ and it follows that $K p \subset V$; thus $K C z \subset V$. Let

$$
H=\{e\} \cup\{y ; y \text { separates } e \text { and } K C z \text { in } X\} .
$$

If $K C z$ is a point, $K$ and $C$ have a fixed point in common, and we are through. Otherwise, $H$ contains no non-cut points of $X$ other than $e$, and $H$ is closed since $X$ is locally connected. This means that $\overline{K x} \subset H$; order $\overline{K x}$ in a standard fashion as follows: $e$ is the first element of $\overline{K x}$ (assuming $e \in \overline{K x})$; if $p, y \in \overline{K x}, p \neq e, y \neq e$, then $p \leqq y$ iff $p=y$ or $p$ separates $e$ from $y$ in $X$. $\leqq$ is a total order on $\overline{K x}$. By virtue of [8], $\overline{K x}$ has a largest element $w ; w$ is a cut point of $X$, and $w$ is evidently fixed under $K$. Further, every point of $C w$ separates $e$ from $C z$. We note that $C_{w}$ is compact and proceed as above to show that $C w$ contains a fixed point of $C$. But this means that $w$ is fixed under $C$. Certainly $w \neq e$, so that $C$ and $K$ have a fixed point, other than $e$, in common.

\section{References}

[1] Hsin Chu, A note on compact transformation groups with a fixed end point, Proc. Amer. Math. Soc. 16 (1965) 581-583.

[2] W. H. Gottschalk and G. A. Hedlund, Topological Dynamics (AMS Colloq. Publ. 36, Providence, R. I., 1955).

[3] William J. Gray, 'Topological transformation groups with a fixed end point', Proc. Amer. Math. Soc. (to appear in 1967).

[4] William J. Gray, 'A note on topological transformation groups with a fixed end point', Pacific J. of Math. (to appear in 1967).

[5] H. C. Wang, 'A remark on transformation group leaving fixed end point,' Proc. Amer. Math. Soc. 3 (1952), 548-549.

[6] A. D. Wallace, 'Group invariant continua', Fund. Math. 36 (1949), $119-124$.

[7] A. D. Wallace, 'Monotone transformations', Duke J. of Math. 9 (1942), 487-506.

[8] A. D. Wallace, 'A fixed point theorem', Bull. Amer. Math. Soc. (1945) 413-416.

[9] R. L. Wilder, Topology of Manifolds (AMS Colloq. Publ. 32, Providence, R. I., 1949).

University of Alabama 